\title{
Reconstruction of inhomogeneous conductivities via the concept of generalized polarization tensors
}

\author{
Habib Ammari ${ }^{a}$, Youjun Deng ${ }^{a, *}$, Hyeonbae Kang ${ }^{\mathrm{b}}$, Hyundae Lee $^{\mathrm{b}}$ \\ ${ }^{a}$ Department of Mathematics and Applications, École Normale Supérieure, 45 Rue d'Ulm, 75005 Paris, France \\ $\mathrm{b}$ Department of Mathematics, Inha University, Incheon 402-751, Republic of Korea
}

Received 19 November 2012; received in revised form 30 July 2013; accepted 31 July 2013

Available online 30 August 2013

\begin{abstract}
This paper extends the concept of generalized polarization tensors (GPTs), which was previously defined for inclusions with homogeneous conductivities, to inhomogeneous conductivity inclusions. We begin by giving two slightly different but equivalent definitions of the GPTs for inhomogeneous inclusions. We then show that, as in the homogeneous case, the GPTs are the basic building blocks for the far-field expansion of the voltage in the presence of the conductivity inclusion. Relating the GPTs to the Neumann-to-Dirichlet (NtD) map, it follows that the full knowledge of the GPTs allows unique determination of the conductivity distribution. Furthermore, we show important properties of the the GPTs, such as symmetry and positivity, and derive bounds satisfied by their harmonic sums. We also compute the sensitivity of the GPTs with respect to changes in the conductivity distribution and propose an algorithm for reconstructing conductivity distributions from their GPTs. This provides a new strategy for solving the highly nonlinear and ill-posed inverse conductivity problem. We demonstrate the viability of the proposed algorithm by preforming a sensitivity analysis and giving some numerical examples.
\end{abstract}

(c) 2013 Elsevier Masson SAS. All rights reserved.

MSC: 35R $30 ; 35 \mathrm{C} 20$

Keywords: Generalized polarization tensors; Inhomogeneous conductivity; Neumann-to-Dirichlet map; Asymptotic expansion; Inverse conductivity problem

\section{Introduction}

There are several geometric and physical quantities associated with shapes such as eigenvalues and capacities [34]. The concept of the generalized polarization tensors (GPTs) is one of them. The notion appears naturally when we describe the perturbation of the electrical potential due to the presence of inclusions whose material parameter (conductivity) is different from that of the background.

\footnotetext{
This work was supported by the ERC Advanced Grant Project MULTIMOD-267184 and the Ministry of Education, Sciences and Technology of Korea through NRF grants Nos. 2009-0090250, 2010-0004091 and 2010-0017532.

* Corresponding author.

E-mail addresses: habib.ammari@ens.fr (H. Ammari), deng@dma.ens.fr (Y. Deng), hbkang@inha.ac.kr (H. Kang), hdlee@inha.ac.kr (H. Lee).
} 
To mathematically introduce the concept of GPTs, we consider the conductivity problem in $\mathbb{R}^{d}, d=2,3$ :

$$
\begin{cases}\nabla \cdot\left(\chi\left(\mathbb{R}^{d} \backslash \bar{\Omega}\right)+k \chi(\Omega)\right) \nabla u=0 & \text { in } \mathbb{R}^{d}, \\ u(x)-h(x)=O\left(|x|^{1-d}\right) & \text { as }|x| \rightarrow \infty .\end{cases}
$$

Here, $\Omega$ is the inclusion embedded in $\mathbb{R}^{d}$ with a Lipschitz boundary, $\chi(\Omega)$ (resp. $\chi\left(\mathbb{R}^{d} \backslash \bar{\Omega}\right)$ ) is the characteristic function of $\Omega$ (resp. $\mathbb{R}^{d} \backslash \bar{\Omega}$ ), the positive constant $k$ is the conductivity of the inclusion which is supposed to be different from the background conductivity $1, h$ is a harmonic function in $\mathbb{R}^{d}$ representing the background electrical potential, and the solution $u$ to the problem represents the perturbed electrical potential. The perturbation $u-h$ due to the presence of the conductivity inclusion $\Omega$ admits the following asymptotic expansion as $|x| \rightarrow \infty$ :

$$
u(x)-h(x)=\sum_{|\alpha|,|\beta| \geqslant 1} \frac{(-1)^{|\beta|}}{\alpha ! \beta !} \partial^{\alpha} h(0) M_{\alpha \beta}(k, \Omega) \partial^{\beta} \Gamma(x),
$$

where $\Gamma$ is the fundamental solution of the Laplacian (see, for example, [7,9]). The building blocks $M_{\alpha \beta}(k, \Omega)$ for the asymptotic expansion (1.2) are called the GPTs. Note that the GPTs $M_{\alpha \beta}(k, \Omega)$ can be reconstructed from the far-field measurements of $u$ by a least-squares method. A stability analysis of the reconstruction is provided in [1]. On the other hand, it is shown in [2] that in the full-view case, the reconstruction problem of GPTs from boundary data has the remarkable property that low order GPTs are not affected by the error caused by the instability of higher-orders in the presence of measurement noise.

The GPTs carry geometric information about the inclusion. For example, the inverse GPT problem holds to be true, namely, the whole set of GPTs, $\left\{M_{\alpha \beta}(k, \Omega):|\alpha|,|\beta| \geqslant 1\right\}$, determines $k$ and $\Omega$ uniquely [6]. The leading order GPT (called the polarization tensor (PT)), $\left\{M_{\alpha \beta}(k, \Omega):|\alpha|,|\beta|=1\right\}$, provides the equivalent ellipse (ellipsoid) which represents overall property of the inclusion $[11,20]$. Moreover, there are important analytical and numerical studies which show that finer details of the shape can be recovered using higher-order GPTs [14,4]. The GPTs even carry topology information of the inclusion [4]. It is also worth mentioning that an efficient algorithm for computing the GPTs is presented in [21].

The notion of GPTs appears in various contexts such as asymptotic models of dilute composites $(c f .[30,32,13])$, low-frequency asymptotics of waves [24], potential theory related to certain questions arising in hydrodynamics [34], biomedical imaging of small inclusions (see [10] and the references therein), reconstructing small inclusions $[27,11,20]$, and shape description [4]. Recently the concept of GPTs finds another promising application to cloaking and electromagnetic and acoustic invisibility. It is shown that the near-cloaking effect of [29] can be dramatically improved by using multi-layered structures whose GPTs vanish up to a certain order [12].

As far as we know, the GPTs have been introduced only for inclusions with homogeneous conductivities or layers with constant conductivities. It is the purpose of this paper to extend the notion of GPTs to inclusions with inhomogeneous conductivities and use this new concept for solving the inverse conductivity problem. We first introduce the GPTs for inhomogeneous inclusions and show that exactly the same kind of far-field asymptotic formula as (1.2) holds. We also prove important properties of the GPTs such as unique determination of Neumann-to-Dirichlet map, symmetry, and positivity. We then provide a sensitivity analysis of the GPTs with respect to changes in the conductivity distribution. We finally propose a minimization algorithm for reconstructing an inhomogeneous conductivity distribution from its high-order GPTs. We carry out a resolution and stability analysis for this reconstruction problem in the linearized case and present numerical examples to show its viability.

The paper is organized as follows. In Section 2 we introduce the GPTs for inhomogeneous conductivity inclusions and prove that they are the building blocks of the far-field expansion of the potential. Section 3 is devoted to the derivation of integral representations of the GPTs. We also establish a relation between the GPTs and the NtD map. In Section 4 we prove important properties of symmetry and positivity of the GPTs and obtain bounds satisfied by their harmonic sums. In Section 5 we perform a sensitivity analysis of the GPTs with respect to the conductivity distribution. We also show that in the linearized case, high-order GPTs capture high-frequency oscillations of the conductivity. In Section 6, we present an algorithm for reconstructing inhomogeneous conductivity distributions from their high-order GPTs. The algorithm is based on minimizing the discrepancy between the computed and measured GPTs. 


\section{Contracted GPTs and asymptotic expansions}

Let $\sigma$ be a bounded measurable function in $\mathbb{R}^{d}, d=2,3$, such that $\sigma-1$ is compactly supported and

$$
\lambda_{1} \leqslant \sigma \leqslant \lambda_{2}
$$

for positive constants $\lambda_{1}$ and $\lambda_{2}$. For a given harmonic function $h$ in $\mathbb{R}^{d}$, we consider the following conductivity problem:

$$
\begin{cases}\nabla \cdot \sigma \nabla u=0 & \text { in } \mathbb{R}^{d}, \\ u(x)-h(x)=O\left(|x|^{1-d}\right) & \text { as }|x| \rightarrow \infty\end{cases}
$$

In this section we derive a full far-field expansion of $(u-h)(x)$ as $|x| \rightarrow \infty$. In the course of doing so, the notion of (contracted) generalized polarization tensors (GPT) appears naturally.

Let $B$ be a bounded domain in $\mathbb{R}^{d}$ with a $\mathcal{C}^{1, \eta}$-boundary $\partial B$ for some $0<\eta<1$. We assume that $B$ is such that

$$
\operatorname{supp}(\sigma-1) \subset B .
$$

Suppose that $B$ contains the origin. Let $H^{s}(\partial B)$, for $s \in \mathbb{R}$, be the usual $L^{2}$-Sobolev space and let $H_{0}^{s}(\partial B):=$ $\left\{\phi \in H^{s}(\partial B) \mid \int_{\partial B} \phi=0\right\}$. For $s=0$, we use the notation $L_{0}^{2}(\partial B)$.

The Neumann-to-Dirichlet (NtD) map $\Lambda_{\sigma}: H_{0}^{-1 / 2}(\partial B) \rightarrow H_{0}^{1 / 2}(\partial B)$ is defined to be

$$
\Lambda_{\sigma}[g]:=\left.u\right|_{\partial B},
$$

where $u$ is the solution to

$$
\begin{cases}\nabla \cdot \sigma \nabla u=0 & \text { in } B, \\ \sigma \frac{\partial u}{\partial v}=g & \text { on } \partial B, \quad\left(\int_{\partial B} u=0\right)\end{cases}
$$

for $g \in H_{0}^{-1 / 2}(\partial B)$. The operator $\Lambda_{1}$ is the NtD map when $\sigma \equiv 1$.

Note that (2.2) is equivalent to

$$
\begin{cases}\nabla \cdot \sigma \nabla u=0 & \text { in } B, \\ \Delta u=0 & \text { in } \mathbb{R}^{d} \backslash \bar{B}, \\ \left.\frac{\partial u}{\partial v}\right|_{+}=\left.\sigma \frac{\partial u}{\partial v}\right|_{-} & \text {on } \partial B, \\ \left.u\right|_{+}=\left.u\right|_{-} & \text {on } \partial B, \\ u(x)-h(x)=O\left(|x|^{1-d}\right) & \text { as }|x| \rightarrow \infty .\end{cases}
$$

Here and throughout this paper, the subscripts \pm indicate the limits from outside and inside $B$, respectively.

Let $\Gamma(x)$ be the fundamental solution to the Laplacian:

$$
\Gamma(x)= \begin{cases}\frac{1}{2 \pi} \ln |x|, & d=2, \\ -\frac{1}{4 \pi}|x|^{-1}, & d=3 .\end{cases}
$$

If $u$ is the solution to (2.2), then by Green's formula we have for $x \in \mathbb{R}^{d} \backslash \bar{B}$

$$
\begin{aligned}
(u-h)(x) & =\left.\int_{\partial B} \Gamma(x-y) \frac{\partial(u-h)}{\partial v}\right|_{+}(y) d s_{y}-\left.\int_{\partial B} \frac{\partial \Gamma(x-y)}{\partial v_{y}}(u-h)\right|_{+}(y) d s_{y} \\
& =\left.\int_{\partial B} \Gamma(x-y) \frac{\partial u}{\partial v}\right|_{+}(y) d s_{y}-\left.\int_{\partial B} \frac{\partial \Gamma(x-y)}{\partial v_{y}} u\right|_{+}(y) d s_{y}
\end{aligned}
$$


where the second equality holds since $h$ is harmonic. Let $g=\left.\sigma \frac{\partial u}{\partial v}\right|_{-}$. Then we have $\left.u\right|_{\partial B}=\Lambda_{\sigma}[g]$ on $\partial B$. Thus we get from the transmission conditions in (2.6) that

$$
(u-h)(x)=\int_{\partial B} \Gamma(x-y) g(y) d s_{y}-\int_{\partial B} \frac{\partial \Gamma(x-y)}{\partial v_{y}} \Lambda_{\sigma}[g](y) d s_{y} .
$$

For $x \in \mathbb{R}^{d} \backslash \bar{B}$, we have

$$
\Lambda_{1}\left(\frac{\partial \Gamma(x-\cdot)}{\partial v_{y}}\right)=\Gamma(x-\cdot)-\frac{1}{|\partial B|} \int_{\partial B} \Gamma(x-y) d s_{y} \quad \text { on } \partial B,
$$

and hence

$$
\int_{\partial B} \frac{\partial \Gamma(x-y)}{\partial v_{y}} \Lambda_{\sigma}[g](y) d s_{y}=\int_{\partial B} \Gamma(x-y) \Lambda_{1}^{-1} \Lambda_{\sigma}[g](y) d s_{y} .
$$

Thus we get from (2.8) and (2.9) that

$$
(u-h)(x)=\int_{\partial B} \Gamma(x-y) \Lambda_{1}^{-1}\left(\Lambda_{1}-\Lambda_{\sigma}\right)[g](y) d s_{y}, \quad x \in \mathbb{R}^{d} \backslash \bar{B} .
$$

Here we have used the fact that $\Lambda_{1}: H_{0}^{-1 / 2}(\partial B) \rightarrow H_{0}^{1 / 2}(\partial B)$ is invertible and self-adjoint:

$$
\left\langle\Lambda_{1}[g], f\right\rangle_{H^{1 / 2}, H^{-1 / 2}}=\left\langle g, \Lambda_{1}[f]\right\rangle_{H^{1 / 2}, H^{-1 / 2}}, \quad \forall f, g \in H_{0}^{-1 / 2}(\partial B),
$$

with $\langle,\rangle_{H^{1 / 2}, H^{-1 / 2}}$ being the duality pair between $H^{-1 / 2}(\partial B)$ and $H^{1 / 2}(\partial B)$.

Suppose that $d=2$. For each positive integer $n$, let $u_{n}^{c}$ and $u_{n}^{s}$ be the solutions to (2.2) when $h(x)=r^{n} \cos n \theta$ and $h(x)=r^{n} \sin n \theta$, respectively. Let

$$
g_{n}^{c}:=\left.\sigma \frac{\partial u_{n}^{c}}{\partial v}\right|_{-} \quad \text { and } \quad g_{n}^{s}:=\left.\sigma \frac{\partial u_{n}^{s}}{\partial v}\right|_{-} \quad \text { on } \partial B .
$$

Since (2.2) is linear, it follows that if the harmonic function $h$ admits the expansion

$$
h(x)=h(0)+\sum_{n=1}^{\infty} r^{n}\left(a_{n}^{c} \cos n \theta+a_{n}^{s} \sin n \theta\right)
$$

with $x=(r \cos \theta, r \sin \theta)$, then we have

$$
g:=\left.\sigma \frac{\partial u}{\partial v}\right|_{-}=\sum_{n=1}^{\infty}\left(a_{n}^{c} g_{n}^{c}+a_{n}^{s} g_{n}^{s}\right),
$$

and hence

$$
(u-h)(x)=\sum_{n=1}^{\infty} \int_{\partial B} \Gamma(x-y)\left(a_{n}^{c} \Lambda_{1}^{-1}\left(\Lambda_{1}-\Lambda_{\sigma}\right)\left[g_{n}^{c}\right](y)+a_{n}^{s} \Lambda_{1}^{-1}\left(\Lambda_{1}-\Lambda_{\sigma}\right)\left[g_{n}^{s}\right](y)\right) d s_{y} .
$$

Note that $\Gamma(x-y)$ admits the expansion

$$
\Gamma(x-y)=\sum_{n=1}^{\infty} \frac{-1}{2 \pi n}\left[\frac{\cos n \theta_{x}}{r_{x}^{n}} r_{y}^{n} \cos n \theta_{y}+\frac{\sin n \theta_{x}}{r_{x}^{n}} r_{y}^{n} \sin n \theta_{y}\right]+C,
$$

where $C$ is a constant, $x=r_{x}\left(\cos \theta_{x}, \sin \theta_{x}\right)$ and $y=r_{y}\left(\cos \theta_{y}, \sin \theta_{y}\right)$. Expansion (2.14) is valid if $|x| \rightarrow \infty$ and $y \in \partial B$. The contracted generalized polarization tensors are defined as follows (see [12]): 


$$
\begin{aligned}
& M_{m n}^{c c}=M_{m n}^{c c}[\sigma]:=\int_{\partial B} r_{y}^{m} \cos m \theta_{y} \Lambda_{1}^{-1}\left(\Lambda_{1}-\Lambda_{\sigma}\right)\left[g_{n}^{c}\right](y) d s_{y}, \\
& M_{m n}^{c s}=M_{m n}^{c s}[\sigma]:=\int_{\partial B} r_{y}^{m} \cos m \theta_{y} \Lambda_{1}^{-1}\left(\Lambda_{1}-\Lambda_{\sigma}\right)\left[g_{n}^{s}\right](y) d s_{y}, \\
& M_{m n}^{s c}=M_{m n}^{s c}[\sigma]:=\int_{\partial B} r_{y}^{m} \sin m \theta_{y} \Lambda_{1}^{-1}\left(\Lambda_{1}-\Lambda_{\sigma}\right)\left[g_{n}^{c}\right](y) d s_{y}, \\
& M_{m n}^{s s}=M_{m n}^{s s}[\sigma]:=\int_{\partial B} r_{y}^{m} \sin m \theta_{y} \Lambda_{1}^{-1}\left(\Lambda_{1}-\Lambda_{\sigma}\right)\left[g_{n}^{s}\right](y) d s_{y} .
\end{aligned}
$$

From (2.13) and (2.14), we get the following theorem.

Theorem 2.1. Let $u$ be the solution to (2.2) with $d=2$. If $h$ admits the expansion (2.12), then we have

$$
(u-h)(x)=-\sum_{m=1}^{\infty} \frac{\cos m \theta}{2 \pi m r^{m}} \sum_{n=1}^{\infty}\left(M_{m n}^{c c} a_{n}^{c}+M_{m n}^{c s} a_{n}^{s}\right)-\sum_{m=1}^{\infty} \frac{\sin m \theta}{2 \pi m r^{m}} \sum_{n=1}^{\infty}\left(M_{m n}^{s c} a_{n}^{c}+M_{m n}^{s s} a_{n}^{s}\right),
$$

which holds uniformly as $|x| \rightarrow \infty$.

In three dimensions, we can decompose harmonic functions as follows:

$$
h(x)=h(0)+\sum_{n=1}^{\infty} \sum_{m=-n}^{n} a_{m n} r^{n} Y_{n}^{m}(\theta, \varphi),
$$

where $(r, \theta, \varphi)$ is the spherical coordinate of $x$ and $Y_{n}^{m}$ is the spherical harmonic function of degree $n$ and of order $m$. Let

$$
g_{m n}=\left.\sigma \frac{\partial u_{m n}}{\partial v}\right|_{-} \text {on } \partial B
$$

where $u_{m n}$ is the solution to (2.2) when $h(x)=r^{n} Y_{n}^{m}(\theta, \varphi)$. It is well-known (see, for example, [36]) that

$$
\Gamma(x-y)=-\sum_{\ell=0}^{\infty} \sum_{k=-\ell}^{\ell} \frac{1}{2 \ell+1} Y_{\ell}^{k}(\theta, \varphi) \overline{Y_{\ell}^{k}\left(\theta^{\prime}, \varphi^{\prime}\right)} \frac{r^{\prime n}}{r^{n+1}},
$$

where $(r, \theta, \varphi)$ and $\left(r^{\prime}, \theta^{\prime}, \varphi^{\prime}\right)$ are the spherical coordinates of $x$ and $y$, respectively. Analogously to Theorem 2.1, the following result holds.

Theorem 2.2. Let $u$ be the solution to (2.2) with $d=3$. If $h$ admits the expansion (2.20), then we have

$$
(u-h)(x)=-\sum_{\ell=1}^{\infty} \sum_{k=-\ell}^{\ell} \sum_{n=1}^{\infty} \sum_{m=-n}^{n} \frac{a_{m n} M_{m n k \ell}}{(2 \ell+1) r^{n+1}} Y_{\ell}^{k}(\theta, \varphi) \quad \text { as }|x| \rightarrow \infty,
$$

where the GPT $M_{m n k \ell}=M_{m n k \ell}[\sigma]$ is defined by

$$
M_{m n k \ell}:=\int_{\partial B} Y_{\ell}^{k}\left(\theta^{\prime}, \varphi^{\prime}\right) r^{\prime n} \Lambda_{1}^{-1}\left(\Lambda_{1}-\Lambda_{\sigma}\right)\left[g_{m n}\right]\left(r^{\prime}, \theta^{\prime}, \varphi^{\prime}\right) d \sigma .
$$

We emphasize that the definitions of contracted GPTs do not depend on the choice of $B$ as long as (2.3) is satisfied. This can be seen easily from (2.19) and (2.23) (see also Section 4). 


\section{Integral representation of GPTs}

In this section, we provide another definition of GPTs which is based on integral equation formulations as in $[28,9]$. Proper linear combinations of GPTs defined in this section coincide with the contracted GPTs defined in the previous section.

Let $N_{\sigma}(x, y)$ be the Neumann function of problem (2.5), that is, for each fixed $z \in B, N_{\sigma}(x, y)$ is the solution to

$$
\left\{\begin{array}{l}
\nabla \cdot \sigma \nabla N(\cdot, z)=-\delta_{z}(\cdot) \quad \text { in } B \\
\left.\sigma \nabla N(\cdot, z) \cdot \nu\right|_{\partial B}=\frac{1}{|\partial B|}, \quad \int_{\partial B} N(x, z) d \sigma(x)=0 .
\end{array}\right.
$$

Then the function $u$ defined by

$$
u(x)=\mathcal{N}_{B, \sigma}[g](x):=\int_{\partial B} N_{\sigma}(x, y) g(y) d s_{y}, \quad x \in B
$$

is the solution to (2.5), and hence

$$
\Lambda_{\sigma}[g](x)=\mathcal{N}_{B, \sigma}[g](x), \quad x \in \partial B .
$$

Let $\mathcal{S}_{B}$ be the single layer potential on $\partial B$, namely,

$$
\mathcal{S}_{B}[\phi](x)=\int_{\partial B} \Gamma(x-y) \phi(y) d s_{y}, \quad x \in \mathbb{R}^{d} .
$$

Let the boundary integral operator $\mathcal{K}_{B}$ (sometimes called the Poincaré-Neumann operator) be defined by

$$
\mathcal{K}_{B}[\phi](x)=\int_{\partial B} \frac{\partial \Gamma}{\partial v_{y}}(x-y) \phi(y) d s_{y} .
$$

It is well-known that the single layer potential $\mathcal{S}_{B}$ satisfies the trace formula

$$
\left.\frac{\partial}{\partial v} \mathcal{S}_{B}[\phi]\right|_{ \pm}=\left( \pm \frac{1}{2} I+\mathcal{K}_{B}^{*}\right)[\phi] \quad \text { on } \partial B
$$

where $\mathcal{K}_{B}^{*}$ is the $L^{2}$-adjoint of $\mathcal{K}_{B}$. We recall that $\lambda I-\mathcal{K}_{B}^{*}$ is invertible on $L_{0}^{2}(\partial B)$ if $|\lambda| \geqslant 1 / 2$ (see, for example, $[26,39,9])$

Identity (2.10) suggests that the solution $u$ to (2.2) may be represented as

$$
u(x)= \begin{cases}h(x)+\mathcal{S}_{B}[\phi](x), & x \in \mathbb{R}^{d} \backslash B, \\ \mathcal{N}_{B, \sigma}[\psi](x)+C, & x \in B\end{cases}
$$

for some densities $\phi$ and $\psi$ on $\partial B$, where the constant $C$ is given by

$$
C=\frac{1}{|\partial B|} \int_{\partial B}\left(h+\mathcal{S}_{B}[\phi]\right) d s .
$$

In view of the transmission conditions along $\partial B$ in (2.2), (3.3) and (3.5), the pair of densities $(\phi, \psi)$ should satisfy

$$
\left\{\begin{array}{l}
-\mathcal{S}_{B}[\phi]+\frac{1}{|\partial B|} \int_{\partial B} \mathcal{S}_{B}[\phi] d s+\Lambda_{\sigma}[\psi]=h-\frac{1}{|\partial B|} \int_{\partial B} h d s, \\
-\left(\frac{1}{2} I+\mathcal{K}_{B}^{*}\right)[\phi]+\psi=\frac{\partial h}{\partial v}
\end{array}\right.
$$

We now prove that the integral equation (3.8) is uniquely solvable. For that, let

$$
\widetilde{\mathcal{S}}_{B}[\phi]:=\mathcal{S}_{B}[\phi]-\frac{1}{|\partial B|} \int_{\partial B} \mathcal{S}_{B}[\phi] d s .
$$


Lemma 3.1. The operator $\mathcal{A}: H^{-1 / 2}(\partial B) \times H_{0}^{-1 / 2}(\partial B) \rightarrow H_{0}^{1 / 2}(\partial B) \times H^{-1 / 2}(\partial B)$ defined by

$$
\mathcal{A}:=\left[\begin{array}{cc}
-\widetilde{\mathcal{S}}_{B} & \Lambda_{\sigma} \\
-\left(\frac{1}{2} I+\mathcal{K}_{B}^{*}\right) & I
\end{array}\right]
$$

is invertible.

As an immediate consequence of Lemma 3.1 we obtain the following theorem.

Theorem 3.1. The solution $u$ to (2.2) can be represented in the form (3.6) where the pair $(\phi, \psi) \in H^{-1 / 2}(\partial B) \times$ $H_{0}^{-1 / 2}(\partial B)$ is the solution to

$$
\mathcal{A}\left[\begin{array}{l}
\phi \\
\psi
\end{array}\right]=\left[\begin{array}{c}
h-\frac{1}{|\partial B|} \int_{\partial B} h d s \\
\left.\frac{\partial h}{\partial \nu}\right|_{\partial B}
\end{array}\right] .
$$

Proof of Lemma 3.1. We first recall the invertibility of $\mathcal{S}_{B}: H^{-1 / 2}(\partial B) \rightarrow H^{1 / 2}(\partial B)$ in three dimensions (see, for instance, [39]). In two dimensions this result is not anymore true. However, using Theorem 2.26 of [9], one can show that in two dimensions there exists a unique $\phi_{0} \in L^{2}(\partial B)$ such that

$$
\int_{\partial B} \phi_{0}=1 \quad \text { and } \quad \widetilde{\mathcal{S}}_{B}\left[\phi_{0}\right]=0 \quad \text { on } \partial B
$$

Then we have

$$
\mathcal{A}\left[\begin{array}{c}
\phi_{0} \\
0
\end{array}\right]=\left[\begin{array}{c}
0 \\
-\left(\frac{1}{2} I+\mathcal{K}_{B}^{*}\right)\left[\phi_{0}\right]
\end{array}\right],
$$

and

$$
\int_{\partial B}\left(\frac{1}{2} I+\mathcal{K}_{B}^{*}\right)\left[\phi_{0}\right] d \sigma=\int_{\partial B} \phi_{0}\left(\frac{1}{2} I+\mathcal{K}_{B}\right)[1] d \sigma=\int_{\partial B} \phi_{0} d \sigma=1 .
$$

Therefore, by replacing $\phi$ with $\phi-\phi_{0} \int_{\partial B} \phi$, it is enough in both the two- and three-dimensional cases to determine uniquely $(\phi, \psi) \in H_{0}^{-1 / 2}(\partial B) \times H_{0}^{-1 / 2}(\partial B)$ satisfying

$$
\mathcal{A}\left[\begin{array}{l}
\phi \\
\psi
\end{array}\right]=\left[\begin{array}{l}
f \\
g
\end{array}\right]
$$

for $(f, g) \in H_{0}^{1 / 2}(\partial B) \times H_{0}^{-1 / 2}(\partial B)$. In fact, if $(f, g) \in H_{0}^{1 / 2}(\partial B) \times H^{-1 / 2}(\partial B)$, then let $C=\frac{1}{|\partial B|} \int_{\partial B} g$ and let $(\phi, \psi)$ be the solution to

$$
\mathcal{A}\left[\begin{array}{l}
\phi \\
\psi
\end{array}\right]=\left[\begin{array}{c}
f \\
g-C\left(\frac{1}{2} I+\mathcal{K}_{B}^{*}\right)\left[\phi_{0}\right]
\end{array}\right] .
$$

It then follows from (3.13) and (3.14) that

$$
\mathcal{A}\left[\begin{array}{c}
\phi-C \phi_{0} \\
\psi
\end{array}\right]=\left[\begin{array}{l}
f \\
g
\end{array}\right] .
$$

We now show that (3.15) is uniquely solvable for a given $(f, g) \in H_{0}^{1 / 2}(\partial B) \times H_{0}^{-1 / 2}(\partial B)$. We first introduce the functional spaces

$$
\begin{aligned}
& H_{\mathrm{loc}}^{1}\left(\mathbb{R}^{d}\right):=\left\{h u \in L^{2}\left(\mathbb{R}^{d}\right), \nabla(h u) \in L^{2}\left(\mathbb{R}^{d}\right), \forall h \in \mathcal{C}_{0}^{\infty}\left(\mathbb{R}^{d}\right)\right\}, \\
& W_{3}\left(\mathbb{R}^{3}\right):=\left\{w \in H_{\mathrm{loc}}^{1}\left(\mathbb{R}^{3}\right): \frac{w}{r} \in L^{2}\left(\mathbb{R}^{3}\right), \nabla w \in L^{2}\left(\mathbb{R}^{3}\right)\right\}
\end{aligned}
$$

and 


$$
W_{2}\left(\mathbb{R}^{2}\right):=\left\{w \in H_{\mathrm{loc}}^{1}\left(\mathbb{R}^{2}\right): \frac{w}{\sqrt{1+r^{2}} \ln \left(2+r^{2}\right)} \in L^{2}\left(\mathbb{R}^{2}\right), \nabla w \in L^{2}\left(\mathbb{R}^{2}\right)\right\},
$$

where $r=|x|$. We also recall that $\Delta$ sets an isomorphism from $W_{d}\left(\mathbb{R}^{d}\right)$ to its dual $W_{d}\left(\mathbb{R}^{d}\right)^{*}$; see, for example, [36].

Observe that it is equivalent to the existence and uniqueness of the solution in $W_{d}\left(\mathbb{R}^{d}\right)$ to the problem (see, for instance, [8, Theorem 2.17])

$$
\begin{cases}\nabla \cdot \sigma \nabla u=0 & \text { in } B, \\ \Delta u=0 & \text { in } \mathbb{R}^{d} \backslash \bar{B}, \\ \left.\sigma \frac{\partial u}{\partial v}\right|_{-}-\left.\frac{\partial u}{\partial v}\right|_{+}=g & \text { on } \partial B, \\ \left.u\right|_{-}-\left.u\right|_{+}=f & \text { on } \partial B, \\ u(x)=O\left(|x|^{1-d}\right) & \text { as }|x| \rightarrow \infty .\end{cases}
$$

The injectivity of $\mathcal{A}$ comes directly from the uniqueness of a solution to (2.6). Since $u$ is harmonic in $\mathbb{R}^{d} \backslash \bar{B}$ and $u(x)=O\left(|x|^{1-d}\right)$ as $|x| \rightarrow \infty$, there exists $\phi \in L_{0}^{2}(\partial B)$ such that

$$
u(x)=\mathcal{S}_{B}[\phi](x), \quad x \in \mathbb{R}^{d} \backslash \bar{B} .
$$

If we set $\psi=\left.\sigma \frac{\partial u}{\partial v}\right|_{-}$, then

$$
\left.u\right|_{-}=\Lambda_{\sigma}[\psi]+C,
$$

where $C=\left.\frac{1}{|\partial B|} \int_{\partial B} u\right|_{-}$. Note that

$$
C=\frac{1}{|\partial B|} \int_{\partial B}\left(\left.u\right|_{+}+f\right)=\frac{1}{|\partial B|} \int_{\partial B} \mathcal{S}_{B}[\phi] .
$$

We now have from (3.19) and (3.21) that

$$
g=\psi-\left(\frac{1}{2} I+\mathcal{K}_{B}^{*}\right)[\phi] .
$$

Furthermore, we have

$$
f=\Lambda_{\sigma}[\psi]+C-\mathcal{S}_{B}[\phi]=\Lambda_{\sigma}[\psi]-\widetilde{\mathcal{S}}_{B}[\phi] .
$$

Thus $(\phi, \psi)$ satisfies (3.15) and the proof is complete.

We can now define the GPTs associated with $\sigma$ using the operator $\mathcal{A}$.

Definition 3.1. Let $\sigma$ be a bounded measurable function in $\mathbb{R}^{d}, d=2,3$, such that $\sigma-1$ is compactly supported and (2.1) holds and let $B$ be a smooth domain satisfying (2.3). For a multi-index $\alpha \in \mathbb{N}^{d}$ with $|\alpha| \geqslant 1$, let $\left(\phi_{\alpha}, \psi_{\alpha}\right) \in$ $H^{-1 / 2}(\partial B) \times H_{0}^{-1 / 2}(\partial B)$ be the solution to

$$
\mathcal{A}\left[\begin{array}{l}
\phi_{\alpha} \\
\psi_{\alpha}
\end{array}\right]=\left[\begin{array}{c}
x^{\alpha}-\frac{1}{|\partial B|} \int_{\partial B} x^{\alpha} d s \\
v \cdot \nabla x^{\alpha}
\end{array}\right] \quad \text { on } \partial B .
$$

For another multi-index $\beta \in \mathbb{N}^{d}$, define the generalized polarization tensors associated with the conductivity distribution $\sigma(x)$ by

$$
M_{\alpha \beta}=M_{\alpha \beta}(\sigma)=\int_{\partial B} x^{\beta} \phi_{\alpha}(x) d s .
$$

Definition 3.1 of the GPTs involves the domain $B$ satisfying (2.3). However, we will show later that GPTs for $\sigma$ (in fact, their harmonic combinations) are independent of the choice of $B$ satisfying (2.3). 
When $|\alpha|=|\beta|=1$, we denote $\mathbf{M}:=\left(M_{\alpha \beta}\right)_{|\alpha|=|\beta|=1}$ and call it the polarization tensor (matrix). Sometimes we write $\mathbf{M}=\left(M_{i j}\right)_{i, j=1}^{d}$.

For a given harmonic function $h$ in $\mathbb{R}^{d}$, let $(\phi, \psi)$ be the solution to (3.11). Since

$$
h(x)=h(0)+\sum_{|\alpha| \geqslant 1} \frac{\partial^{\alpha} h(0)}{\alpha !} x^{\alpha},
$$

we have

$$
\left[\begin{array}{l}
\phi \\
\psi
\end{array}\right]=\sum_{|\alpha| \geqslant 1} \frac{\partial^{\alpha} h(0)}{\alpha !}\left[\begin{array}{l}
\phi_{\alpha} \\
\psi_{\alpha}
\end{array}\right] .
$$

By (3.6) the solution $u$ to (2.2) can be written as

$$
u(x)=h(x)+\sum_{|\alpha| \geqslant 1} \frac{\partial^{\alpha} h(0)}{\alpha !} \mathcal{S}_{B}\left[\phi_{\alpha}\right](x), \quad x \in \mathbb{R}^{d} \backslash B .
$$

Using the Taylor expansion

$$
\Gamma(x-y)=\sum_{|\beta|=0}^{+\infty} \frac{(-1)^{|\beta|}}{\beta !} \partial^{\beta} \Gamma(x) y^{\beta}
$$

which holds for all $x$ such that $|x| \rightarrow \infty$ while $y$ is bounded [9], we obtain the following theorem.

Theorem 3.2. For a given harmonic function $h$ in $\mathbb{R}^{d}$, let $u$ be the solution to (2.2). The following asymptotic formula holds uniformly as $|x| \rightarrow \infty$ :

$$
u(x)-h(x)=\sum_{|\alpha|,|\beta| \geqslant 1} \frac{(-1)^{|\beta|}}{\alpha ! \beta !} \partial^{\alpha} h(0) M_{\alpha \beta} \partial^{\beta} \Gamma(x) .
$$

There is yet another way to represent the solution to (3.11). To explain it, let $\Lambda^{e}$ be the NtD map for the exterior problem:

$$
\Lambda^{e}[g]:=\left.u\right|_{\partial B}-\frac{1}{|\partial B|} \int_{\partial B} u
$$

where $u$ is the solution to

$$
\begin{cases}\Delta u=0 & \text { in } \mathbb{R}^{d} \backslash \bar{B}, \\ \left.\frac{\partial u}{\partial v}\right|_{+}=g & \text { on } \partial B, \\ u(x)=O\left(|x|^{1-d}\right) & \text { as }|x| \rightarrow \infty .\end{cases}
$$

Let $(\phi, \psi)$ be the solution to (3.11). By (3.22), we have

$$
\psi=\left(\frac{1}{2} I+\mathcal{K}_{B}^{*}\right)[\phi]+\left.\frac{\partial h}{\partial v}\right|_{\partial B}=\phi+\left(-\frac{1}{2} I+\mathcal{K}_{B}^{*}\right)[\phi]+\left.\frac{\partial h}{\partial v}\right|_{\partial B} .
$$

On one hand, we obtain from the second identity in (3.29) that $\int_{\partial B} \phi=0$. On the other hand, the first identity in (3.29) says that

$$
\psi=\left.\frac{\partial}{\partial v} \mathcal{S}_{B}[\phi]\right|_{+}+\left.\frac{\partial h}{\partial v}\right|_{\partial B} \quad \text { on } \partial B
$$

and hence

$$
\Lambda^{e}[\psi]=\mathcal{S}_{B}[\phi]-\frac{1}{|\partial B|} \int_{\partial B} \mathcal{S}_{B}[\phi]+\Lambda^{e}\left[\left.\frac{\partial h}{\partial v}\right|_{\partial B}\right] .
$$


Moreover,

$$
\psi=\phi+\left.\frac{\partial}{\partial v} \mathcal{S}_{B}[\phi]\right|_{-}+\left.\frac{\partial h}{\partial v}\right|_{\partial B} \quad \text { on } \partial B,
$$

and therefore,

$$
\Lambda_{1}[\psi]=\Lambda_{1}[\phi]+\mathcal{S}_{B}[\phi]+\left.h\right|_{\partial B}-\frac{1}{|\partial B|}\left(\mathcal{S}_{B}[\phi]+h\right) .
$$

Combining (3.31) and (3.33) with

$$
\Lambda_{\sigma}[\psi]=\mathcal{S}_{B}[\phi]+\left.h\right|_{\partial B}-\frac{1}{|\partial B|}\left(\mathcal{S}_{B}[\phi]+h\right)
$$

in (3.11) yields

$$
\begin{aligned}
& \left(\Lambda_{\sigma}-\Lambda^{e}\right)[\psi]=\left(\Lambda_{1}-\Lambda^{e}\right)\left[\frac{\partial h}{\partial v}\right], \\
& \left(\Lambda_{1}-\Lambda_{\sigma}\right)[\psi]=\Lambda_{1}[\phi] .
\end{aligned}
$$

Thus we readily get

$$
\begin{aligned}
\phi & =\Lambda_{1}^{-1}\left(\Lambda_{1}-\Lambda_{\sigma}\right)\left(\Lambda_{\sigma}-\Lambda^{e}\right)^{-1}\left(\Lambda_{1}-\Lambda^{e}\right)\left[\left.\frac{\partial h}{\partial v}\right|_{\partial B}\right], \\
\psi & =\left(\Lambda_{\sigma}-\Lambda^{e}\right)^{-1}\left(\Lambda_{1}-\Lambda^{e}\right)\left[\left.\frac{\partial h}{\partial v}\right|_{\partial B}\right] .
\end{aligned}
$$

Note that by the uniqueness of a solution to problem (3.18), it is easy to see that $\left(\Lambda_{\sigma}-\Lambda^{e}\right): H_{0}^{-1 / 2}(\partial B) \rightarrow H_{0}^{1 / 2}(\partial B)$ is invertible.

Using (3.34) gives a slightly different but equivalent definition of the GPTs.

Lemma 3.2. For all $\alpha, \beta \in \mathbb{N}^{d}, M_{\alpha \beta}$, defined by (3.25), can be rewritten in the following form:

$$
M_{\alpha \beta}(\sigma)=\int_{\partial B} x^{\beta} \Lambda_{1}^{-1}\left(\Lambda_{1}-\Lambda_{\sigma}\right)\left(\Lambda_{\sigma}-\Lambda^{e}\right)^{-1}\left(\Lambda_{1}-\Lambda^{e}\right)\left[\left.\frac{\partial x^{\alpha}}{\partial v}\right|_{\partial B}\right] d s .
$$

Formula (3.36) shows how to get the GPTs from the NtD maps.

\section{Properties of GPTs}

In this section, we prove important properties for the GPTs. We emphasize that the harmonic sums of GPTs, not individual ones, play a key role. Let $I$ and $J$ be finite index sets. Harmonic sums of GPTs are $\sum_{\alpha \in I, \beta \in J} a_{\alpha} b_{\beta} M_{\alpha \beta}$ where $\sum_{\alpha \in I} a_{\alpha} x^{\alpha}$ and $\sum_{\beta \in J} b_{\beta} x^{\beta}$ are harmonic polynomials.

The following lemma will be useful later.

Lemma 4.1. Let $I$ and $J$ be finite index sets. Let $h_{1}(x):=\sum_{\alpha \in I} a_{\alpha} x^{\alpha}$ and $h_{2}(x):=\sum_{\beta \in J} b_{\beta} x^{\beta}$ be harmonic polynomials and let $u_{1}$ be the solution to (2.2) with $h_{1}(x)$ in the place of $h(x)$. Then,

$$
\sum_{\alpha \in I} \sum_{\beta \in J} a_{\alpha} b_{\beta} M_{\alpha \beta}=\int_{\mathbb{R}^{d}}(\sigma-1) \nabla u_{1} \cdot \nabla h_{2} d x .
$$

Proof. Let $\psi=\sum_{\alpha \in I} a_{\alpha} \psi_{\alpha}$ and $\phi=\sum_{\alpha \in I} a_{\alpha} \phi_{\alpha}$. Then $u_{1}$ is given by

$$
u_{1}(x):= \begin{cases}h_{1}(x)+\mathcal{S}_{B}[\phi](x), & x \in \mathbb{R}^{d} \backslash B, \\ \mathcal{N}_{B, \sigma}[\psi](x)+C, & x \in B .\end{cases}
$$


By (3.24), (3.25), and the integration by parts, we see

$$
\begin{aligned}
\sum_{\alpha \in I} \sum_{\beta \in J} a_{\alpha} b_{\beta} M_{\alpha \beta} & =\int_{\partial B} h_{2}(x) \phi(x) d s_{x} \\
& =\int_{\partial B} h_{2}\left(\left.\frac{\partial \mathcal{S}_{B}[\phi]}{\partial v}\right|_{+}-\left.\frac{\partial \mathcal{S}_{B}[\phi]}{\partial v}\right|_{-}\right) d s_{x} \\
& =\int_{\partial B} h_{2}\left(\psi-\frac{\partial h_{1}}{\partial v}\right) d s_{x}-\left.\int_{\partial B} h_{2} \frac{\partial \mathcal{S}_{B}[\phi]}{\partial v}\right|_{-} d s_{x} \\
& =\int_{\partial B} h_{2}\left(\psi-\frac{\partial h_{1}}{\partial v}\right) d s_{x}-\int_{\partial B} \mathcal{S}_{B}[\phi] \frac{\partial h_{2}}{\partial v} d s_{x} \\
& =\int_{\partial B} h_{2}\left(\psi-\frac{\partial h_{1}}{\partial v}\right) d s_{x}-\int_{\partial B}\left(\Lambda_{\sigma}[\psi]-h_{1}\right) \frac{\partial h_{2}}{\partial v} d s_{x} \\
& =\int_{\partial B}\left(h_{2} \psi-\Lambda_{\sigma}[\psi] \frac{\partial h_{2}}{\partial v}\right) d s_{x} \\
& =\int_{\partial B}\left(\left.h_{2} \sigma \frac{\partial u}{\partial v}\right|_{-}-u \frac{\partial h_{2}}{\partial v}\right) d s_{x} \\
& =\int_{B}(\sigma-1) \nabla h_{2} \cdot \nabla u_{1} d x
\end{aligned}
$$

which concludes the proof.

Identity (4.1) shows in particular that the definition of (harmonic combinations of) the GPTs given in the previous section is independent of the choice of $B$.

\subsection{Symmetry}

We now prove symmetry of GPTs.

Lemma 4.2. Let $I$ and $J$ be finite index sets. For any harmonic coefficients $\left\{a_{\alpha} \mid \alpha \in I\right\}$ and $\left\{b_{\beta} \mid \beta \in J\right\}$, we have

$$
\sum_{\alpha \in I} \sum_{\beta \in J} a_{\alpha} b_{\beta} M_{\alpha \beta}=\sum_{\alpha \in I} \sum_{\beta \in J} a_{\alpha} b_{\beta} M_{\beta \alpha} .
$$

In particular, the first-order GPT, $\mathbf{M}$, is symmetric.

Proof. The symmetry property (4.2) can be easily deduced from the proof of Lemma 4.1. However, we give here a slightly different proof. For doing so, let

$$
h_{1}(x):=\sum_{\alpha \in I} a_{\alpha} x^{\alpha}, \quad h_{2}(x):=\sum_{\beta \in J} b_{\beta} x^{\beta} .
$$

By (3.34), we have

$$
\begin{aligned}
\sum_{\alpha \in I} \sum_{\beta \in J} a_{\alpha} b_{\beta} M_{\alpha \beta} & =\int_{\partial B} h_{2}(x) \phi(x) d s_{x} \\
& =\int_{\partial B} h_{2} \Lambda_{1}^{-1}\left(\Lambda_{1}-\Lambda_{\sigma}\right)\left(\Lambda_{\sigma}-\Lambda^{e}\right)^{-1}\left(\Lambda_{1}-\Lambda^{e}\right)\left[\left.\frac{\partial h_{1}}{\partial v}\right|_{\partial B}\right] \\
& =\int_{\partial B} \frac{\partial h_{2}}{\partial v}\left(\Lambda_{1}-\Lambda_{\sigma}\right)\left(\Lambda_{\sigma}-\Lambda^{e}\right)^{-1}\left(\Lambda_{1}-\Lambda^{e}\right)\left[\left.\frac{\partial h_{1}}{\partial v}\right|_{\partial B}\right] .
\end{aligned}
$$


Since

$$
\left(\Lambda_{\sigma}-\Lambda^{e}\right)^{-1}=\left(\Lambda_{1}-\Lambda^{e}\right)^{-1}+\left(\Lambda_{\sigma}-\Lambda^{e}\right)^{-1}\left(\Lambda_{1}-\Lambda_{\sigma}\right)\left(\Lambda_{1}-\Lambda^{e}\right)^{-1},
$$

we have

$$
\sum_{\alpha \in I} \sum_{\beta \in J} a_{\alpha} b_{\beta} M_{\alpha, \beta}=\int_{\partial B} \frac{\partial h_{2}}{\partial v}\left(\Lambda_{1}-\Lambda_{\sigma}\right)\left[\frac{\partial h_{1}}{\partial v}\right]+\int_{\partial B} \frac{\partial h_{2}}{\partial v}\left(\Lambda_{1}-\Lambda_{\sigma}\right)\left(\Lambda_{\sigma}-\Lambda^{e}\right)^{-1}\left(\Lambda_{1}-\Lambda_{\sigma}\right)\left[\frac{\partial h_{1}}{\partial v}\right] .
$$

Since the NtD maps, $\Lambda_{1}, \Lambda_{\sigma}$, and $\Lambda^{e}$, are self-adjoint, we get (4.2) which concludes the proof.

\subsection{Positivity and bounds}

Let $h(x)=\sum_{\alpha \in I} a_{\alpha} x^{\alpha}$ be a harmonic function in $\mathbb{R}^{d}$ and $u$ be the solution to (2.2). As in the proof of Lemma 4.1, we have

$$
\begin{aligned}
\sum_{\alpha, \beta \in I} a_{\alpha} a_{\beta} M_{\alpha \beta} & =\int_{\partial B}\left(\left.h \sigma \frac{\partial u}{\partial v}\right|_{-}-u \frac{\partial h}{\partial v}\right) d s \\
& =\int_{\partial B}\left(\left.u \sigma \frac{\partial u}{\partial v}\right|_{-}-2(u-h) \frac{\partial h}{\partial v}-h \frac{\partial h}{\partial v}-\left.(u-h) \frac{\partial(u-h)}{\partial v}\right|_{+}\right) d s \\
& =\int_{B}\left(\sigma|\nabla u|^{2}-2 \nabla(u-h) \cdot \nabla h-|\nabla h|^{2}\right)+\int_{\mathbb{R}^{d} \backslash \bar{B}}|\nabla(u-h)|^{2} \\
& =\int_{\mathbb{R}^{d}}\left(\sigma|\nabla(u-h)|^{2}+2(\sigma-1) \nabla(u-h) \cdot \nabla h+(\sigma-1)|\nabla h|^{2}\right) \\
& =\int_{\mathbb{R}^{d}} \sigma\left|\nabla(u-h)+\left(1-\sigma^{-1}\right) \nabla h\right|^{2}+\int_{B} \frac{(\sigma-1)}{\sigma}|\nabla h|^{2} .
\end{aligned}
$$

We can also check the following variational principle:

$$
\sum_{\alpha, \beta \in I} a_{\alpha} a_{\beta} M_{\alpha \beta}=\min _{w \in W_{d}\left(\mathbb{R}^{d}\right)} \int_{\mathbb{R}^{d}} \sigma\left|\nabla w+\left(1-\sigma^{-1}\right) \nabla h\right|^{2}+\int_{B} \frac{(\sigma-1)}{\sigma}|\nabla h|^{2},
$$

where $W_{d}\left(\mathbb{R}^{d}\right)$ is defined by (3.16) and (3.17).

Following the same lines of proof as in [9] for the homogeneous case, we have the following bounds for GPTs.

Theorem 4.1. Let I be a finite index set. Let $\left\{a_{\alpha} \mid \alpha \in I\right\}$ be the set of coefficients such that $h(x):=\sum_{\alpha \in I} a_{\alpha} x^{\alpha}$ is a harmonic function. Then we have

$$
\int_{B} \frac{(\sigma-1)}{\sigma}|\nabla h|^{2} \leqslant \sum_{\alpha, \beta \in I} a_{\alpha} a_{\beta} M_{\alpha \beta} \leqslant \int_{B}(\sigma-1)|\nabla h|^{2} .
$$

Proof. The bound on the left-hand side is obvious since

$$
\min _{w \in W_{d}\left(\mathbb{R}^{d}\right)} \int_{\mathbb{R}^{d}} \sigma\left|\nabla w+\left(1-\sigma^{-1}\right) \nabla h\right|^{2} \geqslant 0 .
$$

By taking $w=0$, we get

$$
\sum_{\alpha, \beta \in I} a_{\alpha} a_{\beta} M_{\alpha \beta} \leqslant \int_{B} \frac{(\sigma-1)^{2}}{\sigma}|\nabla h|^{2}+\int_{B} \frac{(\sigma-1)}{\sigma}|\nabla h|^{2}=\int_{B}(\sigma-1)|\nabla h|^{2},
$$

which concludes the proof. 
The above theorem shows that if $\sigma$ is strictly lager than 1 then the GPTs are positive definite, and they are negative definite if $0<\sigma<1$. Note that optimal bounds on the first-order GPT have been derived in [22,31].

\subsection{GPTs and contracted GPTs}

The contracted GPTs appeared in the asymptotic expansions as in (2.19) and (2.23) while the GPTs appeared in (3.27). By comparing those asymptotic formulas, we obtain the following lemma which relates both quantities.

\section{Lemma 4.3.}

(i) If $r^{n} \cos n \theta=\sum_{|\alpha|=n} a_{\alpha}^{c} x^{\alpha}$ and $r^{n} \sin n \theta=\sum_{|\alpha|=n} a_{\alpha}^{s} x^{\alpha}$ in two dimensions, then

$$
\begin{aligned}
M_{m n}^{c c} & =\sum_{|\alpha|=m,|\beta|=n} a_{\alpha}^{c} a_{\beta}^{c} M_{\alpha \beta}, & M_{m n}^{c s} & =\sum_{|\alpha|=m,|\beta|=n} a_{\alpha}^{c} a_{\beta}^{s} M_{\alpha \beta}, \\
M_{m n}^{s c} & =\sum_{|\alpha|=m,|\beta|=n} a_{\alpha}^{s} a_{\beta}^{c} M_{\alpha \beta}, & M_{m n}^{s s} & =\sum_{|\alpha|=m,|\beta|=n} a_{\alpha}^{s} a_{\beta}^{s} M_{\alpha \beta} .
\end{aligned}
$$

(ii) If $r^{n} Y_{n}^{m}(\theta, \varphi)=\sum_{|\alpha|=n} a_{\alpha}^{m n} x^{\alpha}$ in three dimensions, then

$$
M_{m n k l}=\sum_{|\alpha|=n,|\beta|=l} a_{\alpha}^{m n} a_{\beta}^{k l} M_{\alpha \beta} .
$$

Conversely, any harmonic combination of the GPTs can be recovered from the contracted GPTs.

\subsection{Determination of NtD map}

It is proved in [6] (see also [9, Theorem 4.9]) that the full set of harmonic combinations of GPTs associated with a homogeneous inclusion determines the NtD map on the boundary of any domain enclosing the inclusion, and hence the inclusion. In the case of inhomogeneous conductivity inclusions, the same proof can be easily adapted to obtain the following result.

Theorem 4.2. Let $I$ and $J$ be finite index sets. Let $\sigma_{i}, i=1,2$, be two conductivity distributions with $\operatorname{supp}\left(\sigma_{i}-1\right) \subset \bar{B}$ and satisfying (2.1). If

$$
\sum_{\alpha \in I} \sum_{\beta \in J} a_{\alpha} b_{\beta} M_{\alpha \beta}\left(\sigma_{1}\right)=\sum_{\alpha \in I} \sum_{\beta \in J} a_{\alpha} b_{\beta} M_{\alpha \beta}\left(\sigma_{2}\right)
$$

for any harmonic coefficients $a_{\alpha}$ and $b_{\beta}$, then

$$
\Lambda_{\sigma_{1}}=\Lambda_{\sigma_{2}} \quad \text { on } \partial B \text {. }
$$

Using uniqueness results of the Calderón problems (for example $[38,15]$ ) one can deduce from $(4.6)$ that $\sigma_{1}=\sigma_{2}$ under some regularity assumptions on the conductivities imposed in those results. In two dimensions, uniqueness holds for conductivities in $L^{\infty}[15]$.

\section{Sensitivity analysis for GPTs}

We now consider the sensitivity of the GPTs with respect to changes in the conductivity distribution. Again, we suppose that $\sigma-1$ is compactly supported in a domain $B$. The perturbation of the conductivity $\sigma$ is given by $\sigma+\epsilon \gamma$, where $\epsilon$ is a small positive parameter, $\gamma$ is compactly supported in $B$ and refers to the direction of the changes. The aim of this section is to derive an asymptotic formula, as $\epsilon \rightarrow 0$, for the perturbation

$$
\triangle_{M}:=\sum_{\alpha \in I} \sum_{\beta \in J} a_{\alpha} b_{\beta}\left(M_{\alpha \beta}(\sigma+\epsilon \gamma)-M_{\alpha \beta}(\sigma)\right),
$$

where $\left\{a_{\alpha}\right\}$ and $\left\{b_{\beta}\right\}$ are harmonic coefficients and $I$ and $J$ are finite index sets. 
Let, as above, $h_{1}$ and $h_{2}$ be the harmonic functions given by

$$
h_{1}(x)=\sum_{\alpha} a_{\alpha} x^{\alpha}, \quad h_{2}(x)=\sum_{\beta} b_{\beta} x^{\beta} .
$$

By (3.34) and a direct calculation we obtain

$$
\begin{aligned}
\triangle_{M}= & \int_{\partial B} h_{2} \Lambda_{1}^{-1}\left(\left(\Lambda_{1}-\Lambda_{\sigma+\epsilon \gamma}\right)\left(\Lambda_{\sigma+\epsilon \gamma}-\Lambda^{e}\right)^{-1}-\left(\Lambda_{1}-\Lambda_{\sigma}\right)\left(\Lambda_{\sigma}-\Lambda^{e}\right)^{-1}\right)\left(\Lambda_{1}-\Lambda^{e}\right)\left[\nabla h_{1} \cdot v\right] \\
= & \int_{\partial B} h_{2} \Lambda_{1}^{-1}\left(\left(\Lambda_{\sigma}-\Lambda_{\sigma+\epsilon \gamma}\right)\left(\Lambda_{\sigma+\epsilon \gamma}-\Lambda^{e}\right)^{-1}\right. \\
& \left.\quad+\left(\Lambda_{1}-\Lambda_{\sigma}\right)\left[\left(\Lambda_{\sigma+\epsilon \gamma}-\Lambda^{e}\right)^{-1}-\left(\Lambda_{\sigma}-\Lambda^{e}\right)^{-1}\right]\right)\left(\Lambda_{1}-\Lambda^{e}\right)\left[\nabla h_{1} \cdot \nu\right] \\
= & \int_{\partial B} h_{2} \Lambda_{1}^{-1}\left(\Lambda_{1}-\Lambda^{e}\right)\left(\Lambda_{\sigma}-\Lambda^{e}\right)^{-1}\left(\Lambda_{\sigma}-\Lambda_{\sigma+\epsilon \gamma}\right)\left(\Lambda_{\sigma+\epsilon \gamma}-\Lambda^{e}\right)^{-1}\left(\Lambda_{1}-\Lambda^{e}\right)\left[\nabla h_{1} \cdot v\right] \\
= & \int_{\partial B}\left(\Lambda_{\sigma}-\Lambda_{\sigma+\epsilon \gamma}\right)\left[g_{2}\right] g_{1}^{\epsilon},
\end{aligned}
$$

where

$$
g_{1}^{\epsilon}=\left(\Lambda_{\sigma+\epsilon \gamma}-\Lambda^{e}\right)^{-1}\left(\Lambda_{1}-\Lambda^{e}\right)\left[\nabla h_{1} \cdot \nu\right] \quad \text { and } \quad g_{2}=\left(\Lambda_{\sigma}-\Lambda^{e}\right)^{-1}\left(\Lambda_{1}-\Lambda^{e}\right)\left[\nabla h_{2} \cdot \nu\right] .
$$

Since $\Lambda_{\sigma}$ is self-adjoint, we have

$$
\triangle_{M}=\frac{1}{2} \int_{\partial B}\left(\Lambda_{\sigma}-\Lambda_{\sigma+\epsilon \gamma}\right)\left[g_{2}+g_{1}^{\epsilon}\right]\left(g_{2}+g_{1}^{\epsilon}\right)-\frac{1}{2} \int_{\partial B}\left(\Lambda_{\sigma}-\Lambda_{\sigma+\epsilon \gamma}\right)\left[g_{2}\right] g_{2}-\frac{1}{2} \int_{\partial B}\left(\Lambda_{\sigma}-\Lambda_{\sigma+\epsilon \gamma}\right)\left[g_{1}^{\epsilon}\right] g_{1}^{\epsilon} .
$$

We need the following two lemmas.

Lemma 5.1. If $u_{1}$ and $u_{2}$ are the solutions of $\nabla \cdot\left(\sigma_{1} \nabla u_{1}\right)=0$ and $\nabla \cdot\left(\sigma_{2} \nabla u_{2}\right)=0$ with the Neumann boundary conditions $\sigma_{1} \frac{\partial u_{1}}{\partial v}=g$ and $\sigma_{2} \frac{\partial u_{2}}{\partial v}=g$ on $\partial B$, respectively, then the following identity holds

$$
\int_{\partial B}\left(\Lambda_{\sigma_{2}}-\Lambda_{\sigma_{1}}\right)[g] g d s=\frac{1}{2} \int_{B}\left(\sigma_{1}-\sigma_{2}\right)\left(\left|\nabla\left(u_{1}-u_{2}\right)\right|^{2}+\left|\nabla u_{1}\right|^{2}+\left|\nabla u_{2}\right|^{2}\right) d x .
$$

Proof. The following identity is well-known (see, for instance, [9]):

$$
\int_{B} \sigma_{1}\left|\nabla\left(u_{1}-u_{2}\right)\right|^{2} d x+\int_{B}\left(\sigma_{1}-\sigma_{2}\right)\left|\nabla u_{1}\right|^{2} d x=\int_{\partial B}\left(\Lambda_{\sigma_{2}}-\Lambda_{\sigma_{1}}\right)[g] g d s .
$$

We also have

$$
\int_{B} \sigma_{2}\left|\nabla\left(u_{1}-u_{2}\right)\right|^{2} d x-\int_{B}\left(\sigma_{1}-\sigma_{2}\right)\left|\nabla u_{2}\right|^{2} d x=\int_{\partial B}\left(\Lambda_{\sigma_{1}}-\Lambda_{\sigma_{2}}\right)[g] g d s .
$$

Subtracting those two equalities we obtain (5.3).

Lemma 5.2. There is a constant $C$ such that

$$
\left\|\Lambda_{\sigma_{1}}-\Lambda_{\sigma_{2}}\right\| \leqslant C\left\|\sigma_{1}-\sigma_{2}\right\|_{L^{\infty}(B)} .
$$

Proof. Let $u_{1}$ and $u_{2}$ be the solutions to $\nabla \cdot\left(\sigma_{1} \nabla u_{1}\right)=0$ and $\nabla \cdot\left(\sigma_{2} \nabla u_{2}\right)=0$ with boundary conditions $\sigma_{1} \frac{\partial u_{1}}{\partial v}=g$ and $\sigma_{2} \frac{\partial u_{2}}{\partial v}=g$ on $\partial B$, respectively, and let $h$ be the harmonic function with $\frac{\partial h}{\partial v}=g$ on $\partial B$. Then we have 


$$
\int_{B} \sigma_{1}\left|\nabla u_{1}\right|^{2}=\int_{B} \nabla h \cdot \nabla u_{1} \leqslant \frac{1}{2 \epsilon} \int_{B}|\nabla h|^{2} d x+\frac{\epsilon}{2} \int_{B}\left|\nabla u_{1}\right|^{2} d x,
$$

for any $\epsilon>0$. Choosing $\epsilon=\inf _{B} \sigma_{1}:=\sigma_{1}$ we get

$$
\int_{B}\left|\nabla u_{1}\right|^{2} d x \leqslant \frac{1}{\frac{\sigma}{1}^{2}} \int_{B}|\nabla h|^{2} d x .
$$

Similarly, we get

$$
\int_{B}\left|\nabla u_{2}\right|^{2} d x \leqslant \frac{1}{\underline{\sigma}_{2}^{2}} \int_{B}|\nabla h|^{2} d x,
$$

where $\underline{\sigma_{2}}:=\inf _{B} \sigma_{2}$. It then follows from (5.3) that

$$
\begin{aligned}
\left|\int_{\partial B}\left(\Lambda_{\sigma_{2}}-\Lambda_{\sigma_{1}}\right)[g] g d s\right| & \leqslant \frac{3}{2}\left\|\sigma_{1}-\sigma_{2}\right\|_{L^{\infty}(B)}\left(\int_{B}\left|\nabla u_{1}\right|^{2} d x+\int_{B}\left|\nabla u_{2}\right|^{2} d x\right) \\
& \leqslant \frac{3}{2}\left(\frac{1}{{\underline{\sigma_{1}^{2}}}^{2}}+\frac{1}{{\underline{\sigma_{2}}}^{2}}\right)\left(\int_{B}|\nabla h|^{2} d x\right)\left\|\sigma_{1}-\sigma_{2}\right\|_{L^{\infty}(B)} \\
& \leqslant C\|g\|_{H^{-1 / 2}(\partial B)}^{2}\left\|\sigma_{1}-\sigma_{2}\right\|_{L^{\infty}(B)} .
\end{aligned}
$$

Thus, we obtain (5.4).

With the notation (5.2) in hand, let

$$
g_{1}:=\left(\Lambda_{\sigma}-\Lambda^{e}\right)^{-1}\left(\Lambda_{1}-\Lambda^{e}\right)\left[\nabla h_{1} \cdot v\right],
$$

and let $u_{i}$, for $i=1,2$, be the solution to

$$
\begin{cases}\nabla \cdot\left(\sigma \nabla u_{i}\right)=0 & \text { in } B \\ \sigma \frac{\partial u_{i}}{\partial v}=g_{i} & \text { on } \partial B\end{cases}
$$

Let $u_{1}^{\epsilon}, v_{1}^{\epsilon}$ and $v_{2}^{\epsilon}$ be the solutions to

$$
\begin{aligned}
& \left\{\begin{array}{l}
\nabla \cdot\left(\sigma \nabla u_{1}^{\epsilon}\right)=0 \quad \text { in } B, \\
\sigma \frac{\partial u_{1}^{\epsilon}}{\partial v}=g_{1}^{\epsilon} \quad \text { on } \partial B,
\end{array}\right. \\
& \begin{cases}\nabla \cdot\left((\sigma+\epsilon \gamma) \nabla v_{1}^{\epsilon}\right)=0 & \text { in } B, \\
\sigma \frac{\partial v_{1}^{\epsilon}}{\partial v}=g_{1}^{\epsilon} & \text { on } \partial B,\end{cases}
\end{aligned}
$$

and

$$
\begin{cases}\nabla \cdot\left((\sigma+\epsilon \gamma) \nabla v_{2}^{\epsilon}\right)=0 & \text { in } B \\ \sigma \frac{\partial v_{2}^{\epsilon}}{\partial v}=g_{2} & \text { on } \partial B\end{cases}
$$

Then by Lemma 5.1 we have

$$
\begin{aligned}
\triangle_{M}= & \frac{1}{4} \int_{B} \epsilon \gamma\left(\left|\nabla\left(u_{2}+u_{1}^{\epsilon}-v_{2}^{\epsilon}-v_{1}^{\epsilon}\right)\right|^{2}+\left|\nabla\left(u_{2}+u_{1}^{\epsilon}\right)\right|^{2}+\left|\nabla\left(v_{2}^{\epsilon}+v_{1}^{\epsilon}\right)\right|^{2}\right) d x \\
& -\frac{1}{4} \int_{B} \epsilon \gamma\left(\left|\nabla\left(u_{2}-v_{2}^{\epsilon}\right)\right|^{2}+\left|\nabla u_{2}\right|^{2}+\left|\nabla v_{2}^{\epsilon}\right|^{2}\right) d x-\frac{1}{4} \int_{B} \epsilon \gamma\left(\left|\nabla\left(u_{1}^{\epsilon}-v_{1}^{\epsilon}\right)\right|^{2}+\left|\nabla u_{1}^{\epsilon}\right|^{2}+\left|\nabla v_{1}^{\epsilon}\right|^{2}\right) d x .
\end{aligned}
$$


Lemma 5.2 yields

$$
\left\|u_{1}^{\epsilon}-u_{1}\right\|_{L^{2}(B)}^{2}=O(\epsilon)
$$

and

$$
\left\|v_{j}^{\epsilon}-u_{j}\right\|_{L^{2}(B)}^{2}=O(\epsilon), \quad j=1,2 .
$$

Thus we get

$$
\triangle_{M}=\frac{\epsilon}{2} \int_{B} \gamma\left(\left|\nabla\left(u_{1}+u_{2}\right)\right|^{2}-\left|\nabla u_{1}\right|^{2}-\left|\nabla u_{2}\right|^{2}\right) d x+O\left(\epsilon^{2}\right),
$$

to arrive at the following theorem.

Theorem 5.1. Let I and $J$ be finite index sets. Let $u_{1}$ and $u_{2}$ be the solutions to (5.6). Then we have

$$
\sum_{\alpha \in I} \sum_{\beta \in J} a_{\alpha} b_{\beta} M_{\alpha \beta}(\sigma+\epsilon \gamma)=\sum_{\alpha \in I} \sum_{\beta \in J} a_{\alpha} b_{\beta} M_{\alpha \beta}(\sigma)+\epsilon \int_{B} \gamma \nabla u_{1} \cdot \nabla u_{2} d x+O\left(\epsilon^{2}\right) .
$$

\section{Reconstruction of an inhomogeneous conductivity distribution}

Over the last decades, a considerable amount of work has been dedicated to the inverse conductivity problem. We refer, for instance, to $[18,23]$ and the references therein.

Here, our approach is completely different. We stably recover some important features of inhomogeneous conductivities using their GPTs. It should be emphasized that the GPTs can be obtained from boundary measurements by solving a least-squares problem [1]. The purpose of this section is to illustrate numerically the viability of this finding.

For doing so, we use a least-square approach (see, for instance, [25]). Let $\sigma^{*}$ be the exact (target) conductivity (in two dimensions) and let $y_{m n}:=M_{m n}\left(\sigma^{*}\right)$ (omitting for the sake of simplicity $c$ and $s$ for the superscripts in contracted GPTs). The general approach is to minimize over bounded conductivities $\sigma$ the discrepancy functional

$$
S(\sigma)=\frac{1}{2} \sum_{m+n \leqslant N} \omega_{m n}\left\|y_{m n}-M_{m n}(\sigma)\right\|^{2}
$$

for some finite number $N$ and some well-chosen weights $\omega_{m n}$. The weights $\omega_{m n}$ are used to enhance resolved features of the conductivity as done in $[3,19]$. We solve the above minimization problem using the gradient descent (Landweber) method.

\subsection{Fréchet derivative and an optimization procedure}

Let, again for the sake of simplicity, $M_{m n}(\sigma)=M_{m n}^{c c}(\sigma)$ be the contracted GPTs for a given conductivity $\sigma$. The Fréchet derivative in the direction of $\gamma, M_{m n}^{\prime}(\sigma)[\gamma]$, is defined to be

$$
M_{m n}^{\prime}(\sigma)[\gamma]:=\lim _{\epsilon \rightarrow 0} \frac{M_{m n}(\sigma+\epsilon \gamma)-M_{m n}(\sigma)}{\epsilon} .
$$

From (5.12) we obtain that

$$
M_{m n}^{\prime}(\sigma)[\gamma]=\int_{B} \gamma \nabla u_{n} \cdot \nabla u_{m} d x
$$

where $u_{n}$ and $u_{m}$ are the solutions of

$$
\begin{cases}\nabla \cdot(\sigma \nabla u)=0 & \text { in } B, \\ \sigma \frac{\partial u}{\partial v}=\left(\Lambda_{\sigma}-\Lambda^{e}\right)^{-1}\left(\Lambda_{1}-\Lambda^{e}\right)[\nabla h \cdot v] & \text { on } \partial B,\end{cases}
$$


with $h=r^{n} \cos n \theta$ and $h=r^{m} \cos m \theta$, respectively. Note that if $M_{m n}(\sigma)$ is one of the other contracted GPTs, then $h$ should be changed accordingly.

One can easily see that the adjoint $M_{m n}^{\prime}(\sigma)^{*}$ of $M_{m n}^{\prime}(\sigma)$ is given by

$$
M_{m n}^{\prime}(\sigma)^{*}[c]=c \nabla u_{m} \cdot \nabla u_{n}, \quad c \in \mathbb{R} .
$$

The gradient descent procedure to solve the least-square problem (6.1) reads

$$
\sigma_{k+1}=\sigma_{k}+\sum_{m, n} \omega_{m n} M_{m n}^{\prime}\left(\sigma_{k}\right)^{*}\left[y_{m n}-M_{m n}\left(\sigma_{k}\right)\right] .
$$

In the numerical implementation, the GPTs for the exact conductivity distribution can be computed by using the following formula:

$$
M_{m n}(\sigma)=\int_{\partial B}\left(h_{1}-\tilde{u}_{1}\right) \sigma \frac{\partial u_{2}}{\partial v} d s=\int_{\partial B} h_{1} \sigma \frac{\partial u_{2}}{\partial v} d s-\int_{\partial B} \frac{\partial h_{1}}{\partial v} u_{2} d s,
$$

where $\tilde{u}_{1}$ and $u_{2}$ are the solutions to

$$
\begin{cases}\nabla \cdot\left(\sigma(x) \nabla \tilde{u}_{1}\right)=0 & \text { in } B, \\ \sigma \frac{\partial \tilde{u}_{1}}{\partial v}=\frac{\partial h_{1}}{\partial v} & \text { on } \partial B, \quad\left(\int_{\partial B} \tilde{u}_{1}=0\right),\end{cases}
$$

and

$$
\begin{cases}\nabla \cdot \sigma \nabla u_{2}=0 & \text { in } \mathbb{R}^{d}, \\ u_{2}(x)-h_{2}(x)=O\left(|x|^{1-d}\right), & |x| \rightarrow \infty\end{cases}
$$

respectively. Here, $h_{1}=r^{n} \cos n \theta$ and $h_{2}=r^{m} \cos m \theta$ in two dimensions.

On the other hand, in order to compute $M_{m n}^{\prime}(\sigma)^{*}$ we need to invert the operator $\Lambda_{\sigma}-\Lambda^{e}$. This can be done iteratively. In fact, the least-square solution to

$$
\left(\Lambda_{\sigma}-\Lambda^{e}\right)[g]=f
$$

is given by

$$
g_{k+1}=g_{k}+\omega\left(\Lambda_{\sigma}-\Lambda^{e}\right)\left(f-\left(\Lambda_{\sigma}-\Lambda^{e}\right)\left[g_{k}\right]\right),
$$

where $\omega$ is a positive step-size.

In order to stably and accurately reconstruct the conductivity distribution, we use a recursive approach proposed in [14] (see also $[3,4,19,16]$ ). We first minimize the discrepancy between the first contracted GPTs for $1 \leqslant$ $m, n \leqslant l$. Then we use the result as an initial guess for the minimization between the GPTs for $1 \leqslant m, n \leqslant l+1$. This corresponds to choosing appropriately the weights $\omega_{m n}$ in (6.1). Moreover, we refine the mesh used to compute the reconstructed conductivity distribution every time we increase the number of used contracted GPTs in the discrepancy functional.

\subsection{Resolution analysis in the linearized case}

Let $d=2$ and let $B$ be a disk centered at the origin. Consider the linearized case by assuming that the conductivity $\sigma$ is given by $\sigma=k+\epsilon \gamma$, where $k \neq 1$ is a positive constant and $\epsilon$ is a small parameter. In that case, using Theorem 5.1 together with Lemma 4.3, one can easily see that

$$
M_{m n}(k+\epsilon \gamma)=M_{m n}(k)+\epsilon \int_{B} \gamma \nabla u_{m} \cdot \nabla u_{n} d x+O\left(\epsilon^{2}\right),
$$

with $u_{m}(x)=r^{m} e^{i m \theta}, u_{n}(x)=r^{n} e^{i n \theta}$, and $x=(r, \theta)$. Hence, it follows that

$$
\left(\int_{B} \gamma(r, \theta) r^{m+n-2} e^{i(m+n) \theta} d \theta d r\right)_{1 \leqslant m, n \leqslant N}
$$




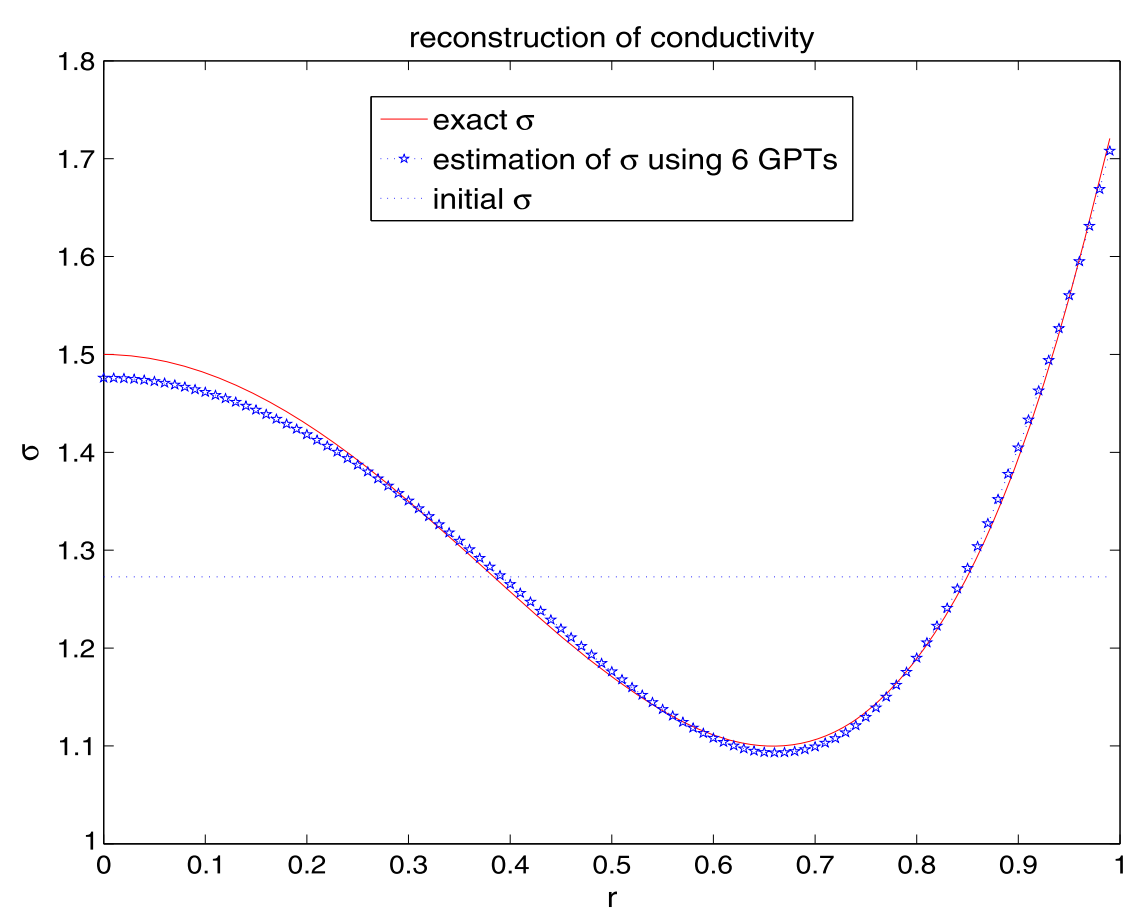

Fig. 1. Reconstructed conductivity distribution.

can be obtained from the contracted GPTs, $M_{m n}$, for $1 \leqslant m, n \leqslant N$. Therefore, the higher is $N$, the better is the angular resolution in reconstructing $\gamma$. On the other hand, it is clear that variations of $\gamma$ that are orthogonal (in the $L^{2}$ sense) to the set of polynomials $\left(r^{m+n-2}\right)_{1 \leqslant m, n \leqslant N}$ cannot be reconstructed from the contracted GPTs $M_{m n}, 1 \leqslant m, n \leqslant N$. Moreover, the reconstruction of $\gamma$ near the origin $(r=0)$ is more sensitive to noise than near the boundary of $B$. This is in accordance with $[5,35]$.

\subsection{Numerical illustration}

In this section, for simplicity we only consider the reconstruction from contracted GPTs of a conductivity distribution which is radially symmetric. Many recent works have been devoted to the reconstruction of radially symmetric conductivities. See, for instance, [17,33,37].

Here we consider the following conductivity distribution:

$$
\sigma=\left(0.3 r^{2}+0.5 r^{3}+6\left(r^{2}-0.5\right)^{2}+3.0\right) / 3.0,
$$

and apply our original approach for recovering $\sigma$ from the contracted GPTs $M_{m n}$, for $m, n \leqslant N$. Since the conductivity distribution $\sigma$ is radially symmetric we have

$$
\begin{array}{ll}
M_{m n}^{c s}=M_{m n}^{s c}=0 & \text { for all } m, n, \\
M_{m n}^{c c}=M_{m n}^{s s}=0 & \text { if } m \neq n,
\end{array}
$$

and $M_{m}:=M_{m m}^{c c}=M_{m m}^{s s}$. We use $M_{1}$ to estimate the constant conductivity which has the same first-order GPT as follows:

$$
\sigma_{0}:=\frac{2|B|+M_{1}}{2|B|-M_{1}}
$$

Then we use $\sigma_{0}$ as an initial guess and apply the recursive approach described below.

Let $k_{*}$ be the last iteration step, and let $\varepsilon_{M}$ and $\varepsilon_{\sigma}$ be discrepancies of GPTs and the conductivities, i.e.,

$$
\varepsilon_{M}:=\sum_{n \leqslant N}\left(y_{n}-M_{n}\left(\sigma_{k_{*}}\right)\right)^{2}, \quad y_{n}:=M_{n}(\sigma) .
$$




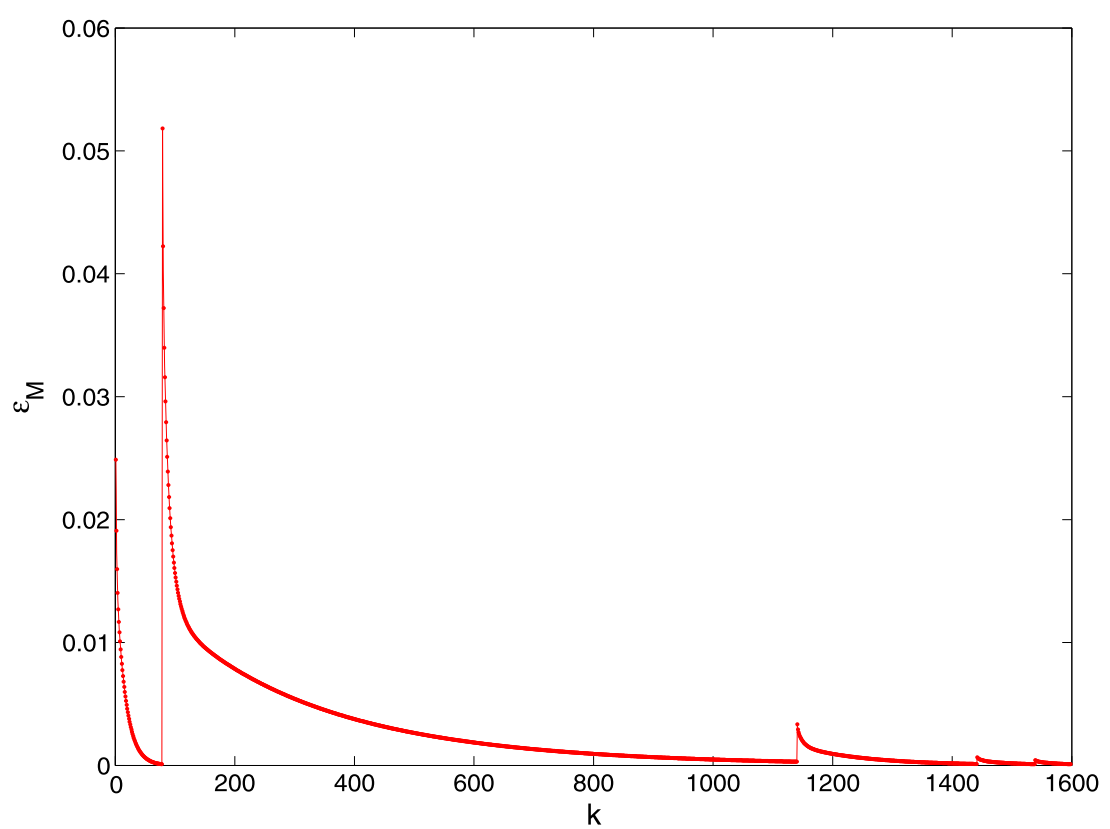

Fig. 2. The convergence history of $\varepsilon_{M}$, where $\mathrm{k}$ is the number of iterations.

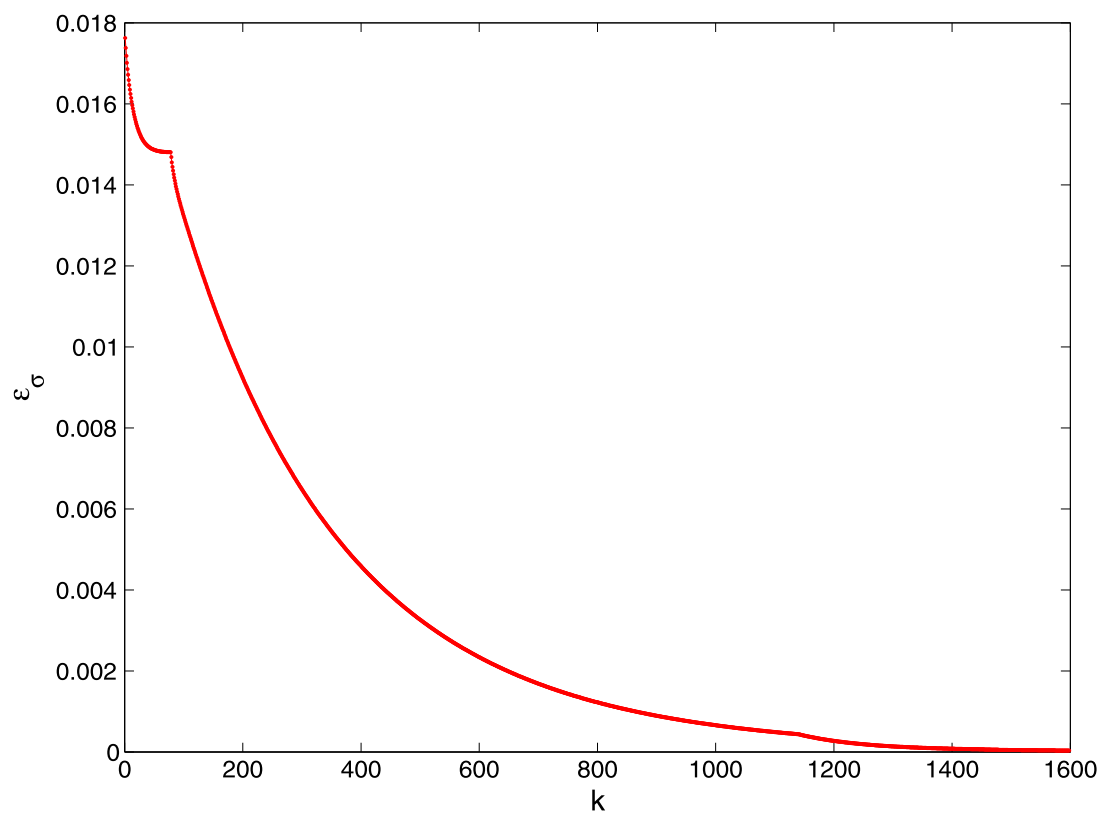

Fig. 3. The convergence history of $\varepsilon_{\sigma}$, where $\mathrm{k}$ is the number of iterations.

( $N$ represents the number of GPTs used) and

$$
\varepsilon_{\sigma}:=\frac{\int_{B}\left(\sigma_{k_{*}}-\sigma\right)^{2}}{\int_{B} \sigma^{2}} .
$$

Fig. 1 shows the reconstructed conductivity distribution using contracted GPTs with $N=6$. In this reconstruction, the errors $\varepsilon_{M}$ and $\varepsilon_{\sigma}$ are given by

$$
\varepsilon_{M}=9.83318 e-005, \quad \varepsilon_{\sigma}=3.5043 e-005,
$$


after 1598 iterations. It should be noted that the conductivity is better reconstructed near the boundary of the inclusion than inside the inclusion itself. Fig. 2 shows how fast $\varepsilon_{M}$ decreases as the iteration proceeds. The sudden jump in the figure happens when we switch the number of GPTs from $N$ to $N+1$. Fig. 3 is for the convergence history of $\varepsilon_{\sigma}$.

\section{Conclusion}

In this paper we have introduced for the first time the notion of GPTs for inhomogeneous conductivity inclusions. The GPTs carry out overall properties of the conductivity distribution. They can be determined from the NtD map. We have established positivity and symmetry properties for the GPTs. We have also analyzed their sensitivity with respect to small changes in the conductivity. We have proposed a recursive algorithm for reconstructing the conductivity from the GPTs and presented a numerical example to show that radially symmetric conductivities can be accurately reconstructed from the GPTs. A numerical study of the use of the GPTs for solving the inverse conductivity problem will be the subject of a forthcoming work. A stability and resolution analysis will be performed. It would also be very interesting to extend the ideas of this paper to the inverse wave medium problems.

\section{References}

[1] H. Ammari, T. Boulier, J. Garnier, W. Jing, H. Kang, H. Wang, Target identification using dictionary matching of generalized polarization tensors, Found. Comput. Math. (2013), in press, arXiv preprint, arXiv:1204.3035.

[2] H. Ammari, T. Boulier, J. Garnier, H. Kang, H. Wang, Tracking of a mobile target using generalized polarization tensors, SIAM J. Imaging Sci. 6 (2013) 1477-1498.

[3] H. Ammari, J. Garnier, H. Kang, M. Lim, K. Sølna, Multistatic imaging of extended targets, SIAM J. Imaging Sci. 5 (2012) 564-600.

[4] H. Ammari, J. Garnier, H. Kang, M. Lim, S. Yu, Generalized polarization tensors for shape description, Numer. Math. (2013), http://dx.doi.org/ 10.1007/s00211-013-0561-5, in press.

[5] H. Ammari, J. Garnier, K. Sølna, Resolution and stability analysis in full-aperture, linearized conductivity and wave imaging, Proc. Am. Math. Soc. 141 (2013) 3431-3446.

[6] H. Ammari, H. Kang, Properties of generalized polarization tensors, Multiscale Model. Simul. 1 (2003) 335-348.

[7] H. Ammari, H. Kang, High-order terms in the asymptotic expansions of the steady-state voltage potentials in the presence of conductivity inhomogeneities of small diameter, SIAM J. Math. Anal. 34 (5) (2003) 1152-1166.

[8] H. Ammari, H. Kang, Reconstruction of Small Inhomogeneities from Boundary Measurements, Lect. Notes Math., vol. 1846, Springer-Verlag, Berlin, 2004.

[9] H. Ammari, H. Kang, Polarization and Moment Tensors with Applications to Inverse Problems and Effective Medium Theory, Appl. Math. Sci., vol. 162, Springer-Verlag, New York, 2007.

[10] H. Ammari, H. Kang, Expansion methods, in: Handbook of Mathematical Mehtods of Imaging, Springer, 2011, pp. 447-499.

[11] H. Ammari, H. Kang, E. Kim, M. Lim, Reconstruction of closely spaced small inclusions, SIAM J. Numer. Anal. 42 (2005) $2408-2428$.

[12] H. Ammari, H. Kang, H. Lee, M. Lim, Enhancement of near cloaking using generalized polarization tensors vanishing structures. Part I: The conductivity problem, Commun. Math. Phys. 317 (2013) 485-502.

[13] H. Ammari, H. Kang, K. Touibi, Boundary layer techniques for deriving the effective properties of composite materials, Asymptot. Anal. 41 (2005) 119-140.

[14] H. Ammari, H. Kang, M. Lim, H. Zribi, The generalized polarization tensors for resolved imaging. Part I: Shape reconstruction of a conductivity inclusion, Math. Comput. 81 (2012) 367-386.

[15] K. Astala, L. Päivärinta, Calderón's inverse conductivity problem in the plane, Ann. Math. 163 (2006) 265-299.

[16] G. Bao, S. Hou, P. Li, Recent studies on inverse medium scattering problems, Lect. Notes Comput. Sci. Eng. 59 (2007) $165-186$.

[17] J. Bikowski, K. Knudsen, J.L. Mueller, Direct numerical reconstruction of conductivities in three dimensions using scattering transforms, Inverse Probl. 27 (2011), Article 015002.

[18] L. Borcea, Electrical impedance tomography, Inverse Probl. 18 (2002) R99-R136.

[19] L. Borcea, G. Papanicolaou, F.G. Vasquez, Edge illumination and imaging of extended reflectors, SIAM J. Imaging Sci. 1 (2008) $75-114$.

[20] M. Brühl, M. Hanke, M.S. Vogelius, A direct impedance tomography algorithm for locating small inhomogeneities, Numer. Math. 93 (4) (2003) 635-654.

[21] Y. Capdeboscq, A.B. Karrman, J.-C. Nédélec, Numerical computation of approximate generalized polarization tensors, Appl. Anal. 91 (2012) $1189-1203$

[22] Y. Capdeboscq, M.S. Vogelius, A general representation formula for the boundary voltage perturbations caused by internal conductivity inhomogeneities of low volume fraction, Math. Model. Numer. Anal. 37 (2003) 159-173.

[23] M. Cheney, D. Isaacson, J.C. Newell, Electrical impedance tomography, SIAM Rev. 41 (1999) 85-101.

[24] G. Dassios, R.E. Kleinman, On Kelvin inversion and low-frequency scattering, SIAM Rev. 31 (1989) 565-585.

[25] H.W. Engl, M. Hanke, A. Neubauer, Regularization of Inverse Problems, Kluwer, Dordrecht, 1996.

[26] G.B. Folland, Introduction to Partial Differential Equations, Princeton University Press, Princeton, NJ, 1976.

[27] A. Friedman, M. Vogelius, Identification of small inhomogeneities of extreme conductivity by boundary measurements: a theorem on continuous dependence, Arch. Ration. Mech. Anal. 105 (1989) 299-326. 
[28] H. Kang, J.K. Seo, Recent progress in the inverse conductivity problem with single measurement, in: Inverse Problems and Related Fields, CRC Press, Boca Raton, FL, 2000, pp. 69-80.

[29] R.V. Kohn, H. Shen, M.S. Vogelius, M.I. Weinstein, Cloaking via change of variables in electric impedance tomography, Inverse Probl. 24 (2008), Article 015016.

[30] S.M. Kozlov, On the domain of variations of added masses, polarization and effective characteristics of composites, J. Appl. Math. Mech. 56 (1992) 102-107.

[31] R. Lipton, Inequalities for electric and elastic polarization tensors with applications to random composites, J. Mech. Phys. Solids 41 (1993) 809-833.

[32] G.W. Milton, The Theory of Composites, Cambridge Monogr. Appl. Comput. Math., Cambridge University Press, 2001.

[33] L.J. Mueller, S. Siltanen, Direct reconstructions of conductivities from boundary measurements, SIAM J. Sci. Comput. 24 (2003) $1232-1266$.

[34] G. Pólya, G. Szegö, Isoperimetric Inequalities in Mathematical Physics, Ann. Math. Stud., vol. 27, Princeton University Press, Princeton, NJ, 1951.

[35] S. Nagayasu, G. Uhlmann, J.-N. Wang, Depth dependent stability estimates in electrical impedance tomography, Inverse Probl. 25 (2009), Article 075001.

[36] J.-C. Nédélec, Acoustic and Electromagnetic Equations. Integral Representations for Harmonic Problems, Appl. Math. Sci., vol. 144, SpringerVerlag, New York, 2001.

[37] S. Siltanen, J.L. Mueller, D. Isaacson, Reconstruction of high contrast 2-D conductivities by the algorithm of A. Nachman, in: Contemp. Math., vol. 278, Amer. Math. Soc., Providence, RI, 2001, pp. 241-254.

[38] J. Sylvester, G. Uhlmann, A global uniqueness theorem for an inverse boundary value problem, Ann. Math. 125 (1987) 153-169.

[39] G.C. Verchota, Layer potentials and boundary value problems for Laplace's equation in Lipschitz domains, J. Funct. Anal. 59 (1984) $572-611$. 